\title{
HLA Mismatch Count
}

National Cancer Institute

\section{Source}

National Cancer Institute. HLA Mismatch Count. NCI Thesaurus. Code C128933.

The determination of the total number of mismatches between the study subject and his or her donor for the human leukocyte antigens (HLA). 